\title{
Cerebral Activation during Anal and Rectal Stimulation
}

\author{
M. Lotze,* B. Wietek,† N. Birbaumer,*ł J . Ehrhardt,* W. Grodd,§ and P. Enck† \\ *Institute of Medical Psychology and Behavioral Neurobiology, †Department of General Surgery, and §Section of Experimental Magnetic \\ Resonance of the CNS, Department of Neuroradiology, University of Tübingen, D-72074 Tübingen, Germany; \\ and ¥Department of General Psychology, University of Padua, Padua, I taly
}

Received J anuary 2, 2001

\begin{abstract}
While the rectum is innervated by visceral afferents, the anal canal is innervated by the somatosensory pudendal nerve. The representation of these two central domains of intestinal sensations in the human brain is largely unknown. Nonpainful pneumatic stimulation of the anal canal and the distal rectum using event-related functional magnetic resonance imaging (fMRI) was performed in eight healthy subjects. Subjective scaling of sensations revealed no differences in unpleasantness and pain during both stimuli. Both types of stimuli revealed fMRI activation in secondary somatosensory, insula, cingular gyrus, left inferior parietal, and right orbitofrontal cortex. Anal stimulation resulted in additional activation of primary sensory and motor cortex, supplementary motor area, and left cerebellum. We concluded that viscerorectal and somatosensory anal stimulation predominantly differ in their primary sensory activation and additional activation in motor areas. This motor response following aversive somatosensory stimuli may be caused by a reflexive avoidance reaction which is not observed after the more diffuse experienced visceral stimulation. 02001 Academic Press

Key Words: visceral; rectal; anal; somatosensory; fMRI.
\end{abstract}

\section{INTRODUCTION}

Perception of information from the anorectum is crucial for maintenance of continence (Whitehead et al., 1981) and is frequently interrupted or blocked in patients with incontinence due to traumatic spinal cord injury, systemic diseases, and neurological deficits. Increased sensibility of this gastrointestinal compartment-also resulting in urge and incontinence-is the major pathomechanism of functional bowel disorders such as the irritable bowel syndrome but may also be a consequence of inflammatory diseases such as ulcerative colitis (Bielefeldt et al., 1990).

Two major perceptive discriminative functions are involved: the rectal sensing of its filling and the anal identification of content and consistency. This discrimination is achieved by two distinctly different innerva- tions: The rectum is innervated by autonomic nerves with sympathetic fibers arising from the lumbar level of the spinal cord (fifth lumbar root) with their postganglionic fibers reaching the anal canal (internal anal sphincter) via the hypogastric and pelvic plexus, while parasympathetic fibers originate from the second to the fourth spinal root. In addition, the internal sphincter muscle receives autonomic input from the myenteric plexus of the rectum (enteric nervous system). In contrast, the anal canal receives sensory and motor projections through the pudendal nerve, which leave the spinal cord at the level of the second to the fourth spinal root bilaterally. The anal canal has a rich supply of nerve endings at the level of the anal skin, while the rectum senses mechanical distention through mechanoreceptors deep in-between the muscle layers of the gut wall. In consequence, rectal perception has a high threshold and is diffuse in localization (Goligher et al., 1951), while anal sensation is precise in localization, possesses a low threshold, and allows precise discrimination (Duthie et al., 1963). While it is conceivable that such differences in peripheral processing of sensory information would result in differences in cortical processing of this information, this has not been investigated for the anorectum so far. It is, however, known from the similar innervation pattern of the human bladder that cortical representation of sensations resulting in micturition and voiding resembles both somatosensory and somatomotor components (primary somatosensory cortex, parietal operculum, cerebellum, premotor cortex) on the one hand and midbrain areas involved in processing of the aversive or emotional components of sensations (cingular gyrus, hypothalamus) on the other hand (Nour et al., 2000; Athwal et al., 2001). Consequently, we would expect a similar network processing sensory information from the anorectum.

Imaging studies of central processing of sensations arising from the rectum have rarely been undertaken and, if so, predominantly for the evaluation of experimental visceral pain in functional bowel disorders (Silvermann et al., 1997; Mertz et al., 2000). These authors 


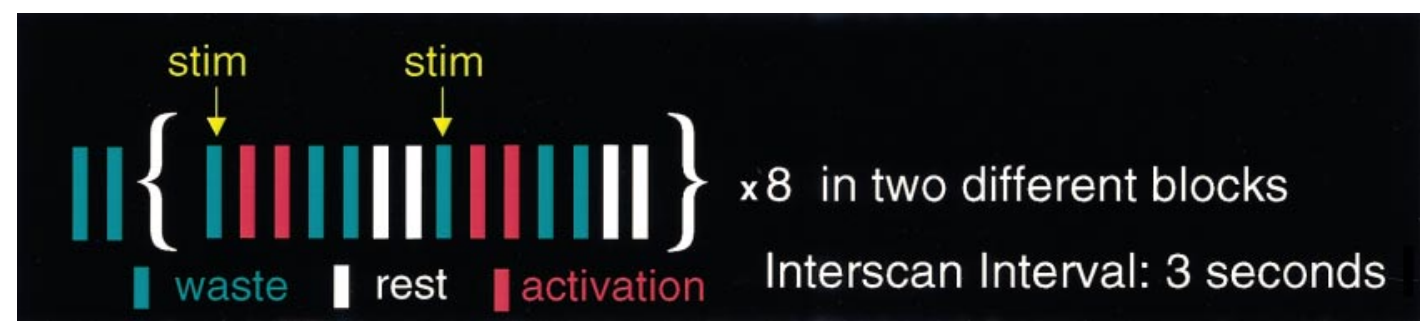

FIG. 1. Event-related experimental design showing onset of pneumatic stimuli and fMRI measurement with 32 measurements per condition. Activated images (red bars) were selected 3 to $9 \mathrm{~s}$ after stimulus onset and compared to rest (white; 15-21 s after stimulus).

identified the insular cortex and the anterior cingular gyrus (ACG) as the major areas of activation following rectal distention, together with the prefrontal cortex (PFC). Whereas the ACG has been shown to be involved in the gating of pain, more posteriorly located areas of the cingular gyrus (CG) encode for unpleasantness of stimuli (Tölle et al., 1999). Furthermore, painful stimulation may additionally involve limbic and thalamic areas (e.g., Di Piero et al., 1994; Coghill et al., 1999). Therefore, the findings of Silverman et al. (1997) and Mertz et al. (2000) may not necessarily be specific for visceral perception but mainly describe areas involved in pain perception. Visceral perception should, therefore, be compared to somatosensory perception by investigating activation maps in response to nonpainful stimuli in neighboring body compartments to minimize effects of interfering variables.
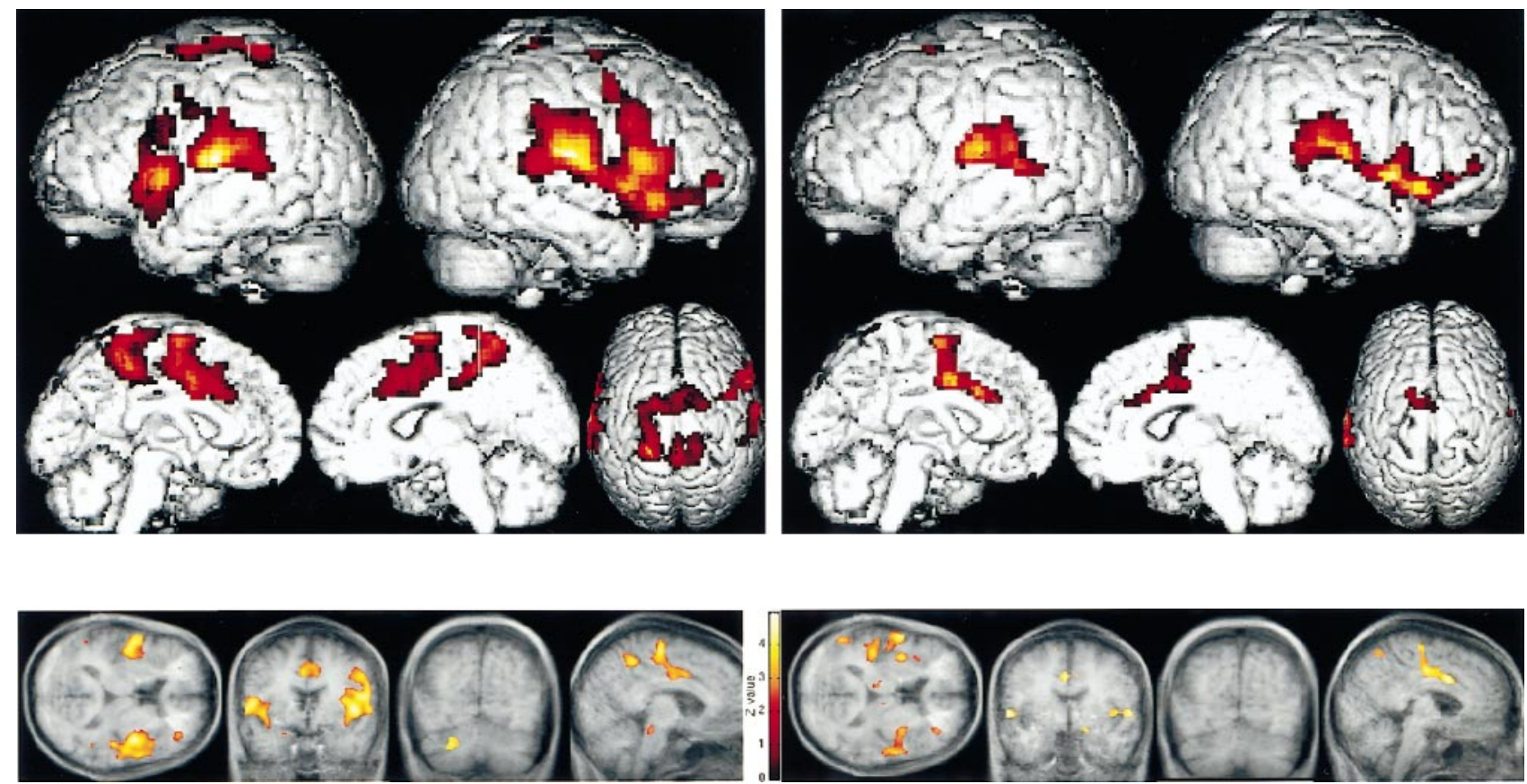

FIG. 2. Activation sites evaluated after group analysis. Left: Anal stimulation. Right: Rectal stimulation. Top: Activation map with an intensity cut-off of $\mathrm{P}<0.01$ (Bonferroni correction of $\mathrm{P}<0.05$ ) projected on the SPM 96 single subject rendered brain. Bottom: Significant activation without a Bonferroni correction (intensity cut-off of $\mathrm{P}<0.01$ ) projected on the average of T1-weighted anatomy of all subjects. Anal stimulation showed significant activation in bilateral insula, right orbitofrontal, cingular gyrus, left cerebellar hemisphere, and periaqueductal gray. Rectal stimulation revealed activation in the insula, orbitofrontal lobe, thalamus (n.s.), and cingular gyrus. 

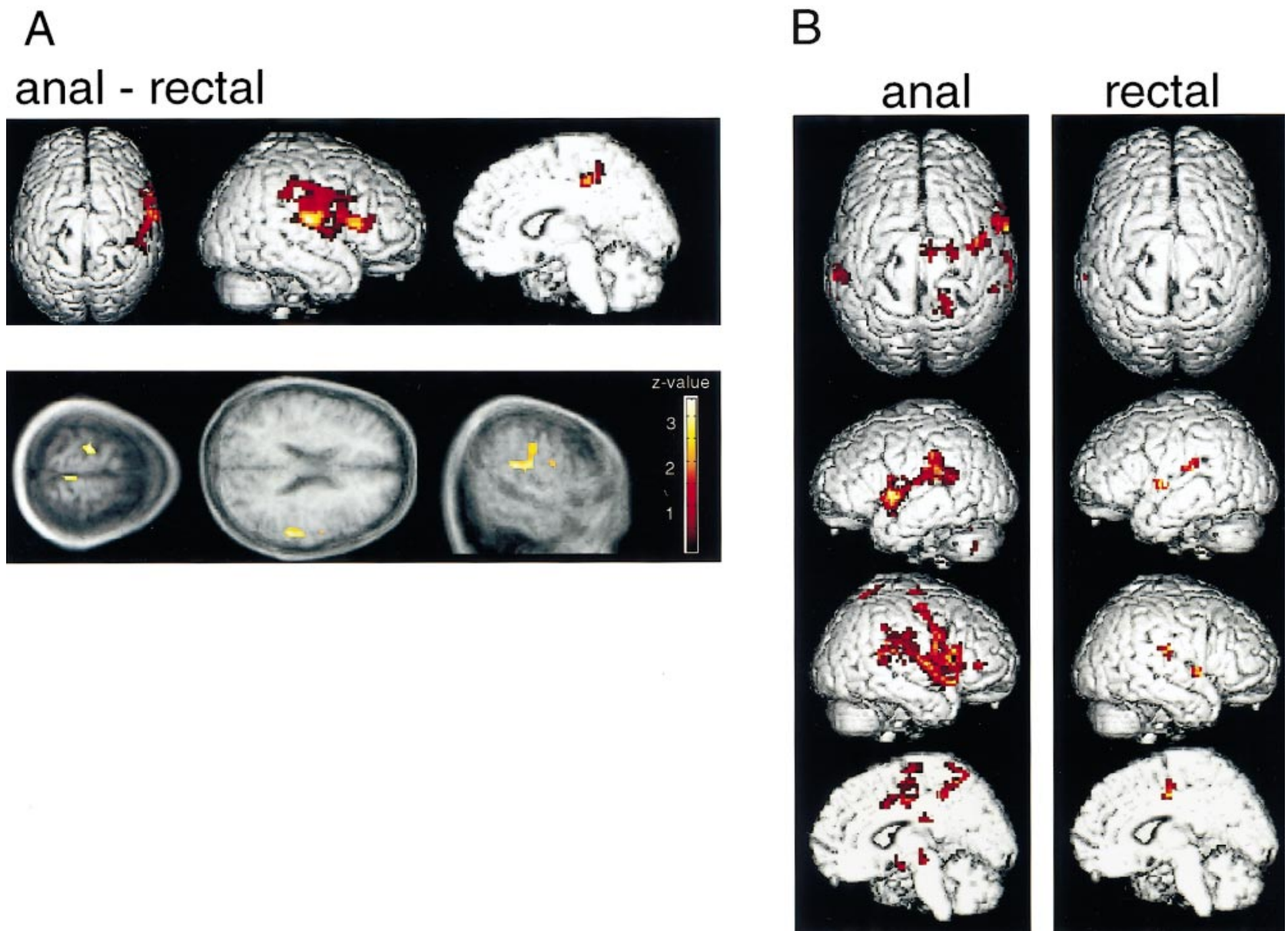

FIG. 3. (A) Direct statistical comparison between anal minus rectal representation maps. Top: Activation map with an intensity cut-off of $\mathrm{P}<0.05$ (Bonferroni correction $\mathrm{P}<0.05$ ) projected on the SPM 96 single subject rendered brain. Right hemispheric S2, BA 44, and activation in the posterior-medial cingular gyrus are observed. Bottom: Significant activation within different regions without a Bonferroni correction (intensity cut-off of $\mathrm{P}<0.01$ ) projected on the average of T1-weighted anatomy of all subjects. Left: $\mathrm{M} 1$ activation in the interhemispheric fissure and left. Middle: I nferior S1 and S2. Right: I nferior right BA 44 and S2. (B) A conjunction analysis of the activation maps of all subjects for each stimulus type ( $<<0.01$, uncorrected) revealed activation of the right $\mathrm{M} 1 / \mathrm{S} 1$, the SMA, the right premotor cortex, the left cerebellar hemisphere, the bilateral insula and S2, and the medial cingulate gyrus for anal stimuli and bilateral insula, the bilateral S2 and the medial cingulate gyrus activation for the rectal stimuli.

ing attentive and emotional aspects. With rectal stimulation we expected activation of the S2 cortex, again indicative of the sensory-discriminative aspect of sensation, and nonspecific activation of the cingular gyrus and the PFC, as well as-to a minor degree-limbic structures. Furthermore, we were interested in the representation sites of the cerebellum since its somatotopic organization (Grodd et al., 2001) has not yet included anal and rectal representations, although left superior medial cerebellar activation was observed during visceromotor functions (Hamdy et al., 1999).

\section{METHODS}

\section{Subjects and Procedure}

Eight healthy subjects (four female and four male; average age 37.3 years, ranging from 28 to 54 years) who had no history of anal or rectal complications were included in the study. They were asked to evacuate their rectum prior to the experiment. A double-balloon catheter was introduced into the rectum so that one balloon was positioned at approximately $15 \mathrm{~cm}$ from the anal verge in the distal rectum and the second balloon was positioned in the anal canal; to ensure stability the probe was fixed to the buttocks.

Pneumatic stimulation elicited feelings of gas and stool pressure. Air inflation of the balloon was performed manually by a physician experienced with anal and rectal manometry (B.W.). To evoke feelings of discomfort but avoid painful stimulation, the inflation of the balloon was individually adjusted using a visual anal ogue score (0, not unpleasant/not painful; 10, maximal unpleasant/unbearable painful). For rectal stimulation, the balloon was preinflated with $100 \mathrm{ml}$ prior 
to the recording, a condition to which all subjects adapted rapidly; this volume served as baseline. Additional volumes between 100 and $250 \mathrm{ml}$ (average 173.6 $\mathrm{ml}$; SD $45.0 \mathrm{ml}$ ) were injected for the activation period. For stimulation of the anal canal, individual volumes between 7.5 and $25 \mathrm{ml}$ (mean $15.5 \mathrm{ml}$, SD $7.7 \mathrm{ml}$ ) were injected into the balloon for activation, but during the rest condition of the anal stimulation sequence the balloon was completely deflated. For each of the two stimulus conditions a stimulus was presented for approx 3 s every 21 s 32 times.

The study was approved by the ethics committee of the Medical School of the University of Tübingen.

\section{Data Acquisition}

fMRI of the whole brain was acquired with a 1.5-T tomograph (Siemens Vision) using echo planar imaging (28 axial slices of $4 \mathrm{~mm}$ slice thickness, 64 matrix, acquisition time $3 \mathrm{~s}$ ). Three hundred thirty-six images were measured for each subject in an event-related design. Additionally, a T1-weighted anatomy 3D dataset containing 128 sagittal slices (effective thickness $1.5 \mathrm{~mm}$; matrix $224 \times 256$; field of view $250 \mathrm{~mm}$; TR 9.7 ms) was measured. I mages were acquired with an improved storage system described by Klose et al. (1999).

\section{Statistical Evaluation}

The fMRI data were evaluated with the Statistical Parametric Mapping program (SPM96; Wellcome Department of Neurology, London). The scans of each individual were realigned to each other to correct for interscan movement artifacts. One subject was excluded from further analysis because he showed movement artifacts larger than $2.5 \mathrm{~mm}$. The echo planar images of each subject were coregistered to the anatomical datasets after manually defining the anterior commissure as a reference point. We performed group statistics using nonlinear normalization $(3 \times 3 \times 3$ $\mathrm{mm}$ ) of the individual dataset to the SPM template. The normalized data were smoothed with a Gaussian filter of $8 \mathrm{~mm}$. Statistics were performed using a fixedeffect model. Statistically significant differences between stimulation and rest were assessed with the delayed boxcar model using t statistics which were then converted to $Z$ scores. I mages from 3 to $9 \mathrm{~s}$ after onset of stimulation (two images; see Fig. 1), which were assumed to be activated, were compared to rest (two images; 15-21 s after the stimulus onset), and for each subject a total of 32 scans contributed to the statistic comparison. We used a statistical threshold of $\mathrm{P}<0.01$ and an additional Bonferroni correction ( $\mathrm{P}<$ 0.05; correction for multiple testing). Direct comparison between different conditions was performed with a height threshold of $\mathrm{P}<0.05$, corrected for the whole volume $(P<0.05)$, evaluating interactions between both conditions ((anal baseline) - (rectal-baseline)). To evaluate smaller activation sites within the basal ganglia, the thalamus, and the cerebellum, no additional Bonferroni correction was used.

The resulting activation sites were described by their intensity with Z values (Z). Location was indicated by coordinates within the SPM reference system, by anatomical structures, and by Brodmann's areas (described by Talairach and Tournoux, 1988).

\section{RESULTS}

The unpleasantness ratings of the subjects revealed a score of 5.24 (SD 2.36) during rectal and 4.76 (SD 2.23) during anal stimulation (no difference in t test: $\mathrm{t}(6)=0.46 ;$ n.s.). No stimulation was perceived as painful (average score 0.30). The event-related design enabled an individual mapping of the cerebral regions.

Somatosensory anal stimulation (see Fig. 2 and Table 1) revealed significant activation in S2 bilateral ( $Z$ : left, 7.46; right, 7.62), insula bilateral (Z: left, 4.38; right, 5.24), predominantly left S1 (Z: left, 6.12; right, 4.16), and left MI (Z: 4.08) centered at the left homuncular trunk representation area. Additional activation was observed in the pre-SMA (Z: 5.19), right BA 44 (Z: 5.94), medial CG (Z: 5.01), left inferior parietal lobe ( $Z$ : 4.32), and right orbitofrontal cortex (BA 11 and 47; Z: 4.01). Non-volume-corrected statistics revealed activation in left amygdala (Z: 3.18), periaqueductal gray (Z: 3.12), and left posterior cerebellar lobe (Z: 3.17).

Visceral rectal stimulation showed activation in $\mathrm{S} 2$ bilateral (Z: left, 5.88; right, 4.63), insula bilateral left, 3.43; right, (Z: 4.19), CG (Z: 4.40), bilateral inferior parietal lobe (Z: left, 3.94; right, 4.22), and right or bitofrontal cortex (BA 11 and 47; Z: 4.18). Bilateral thalamus was activated but failed significance with and without volume correction (Z: 2.95; n.s.). No activation was observed in S1, M1, SMA, or any other region.

Direct calculation of activation maps during anal stimulation minus rectal with Bonferroni correction revealed higher activation in the medial CG ( $Z$ : 3.14), in right $S 2$ (Z: 3.78), and in right $B A 44$ (Z: 3.50; see Fig. 3A, top, and Table 1 ). Without volume correction smaller additional activation sites in interhemispheric MI (Z: 3.41), in S1 at the inferior left representational site ( $Z$ : 3.26), and in the SMA ( $Z$ : 3.27; see Fig. 3A, bottom) were observed. The calculation "rectal minus anal" showed no significant differences.

A conjunction analysis was performed to add information on the reproducibility of the data. This analysis (see Fig. 3B) confirmed that anal stimulation activates also motor areas. In this analysis rectal stimulation revealed activations only in the bilateral insula and S2 and in the medial cingulate gyrus.

Individual data indicate large variation in response to the stimuli. Low stimulus intensity resulted in low significance of activation sites as in subject 6 , who tolerated only low amounts of air inflation (100 ml 
TABLE 1

Activation Sites during Anal and Rectal Stimulation Compared to Rest and by Calculation of the Interaction of Anal Minus Rectal Stimulation (Right Column)

\begin{tabular}{|c|c|c|c|c|c|c|}
\hline \multirow[b]{2}{*}{ Region } & \multicolumn{2}{|c|}{ Anal } & \multicolumn{2}{|c|}{ Distal rectum } & \multicolumn{2}{|c|}{ Anal minus rectal } \\
\hline & $Z$ value & Coordinates & $Z$ value & Coordinates & $Z$ value & Coordinates \\
\hline M1 (BA 4) & 4.08 & $-24 ;-9 ; 66$ & - & - & - & - \\
\hline S1 (BA 1-3) L & 6.12 & $-18 ;-39 ; 69$ & - & - & - & - \\
\hline S1 (BA 1-3) R & 4.16 & $3,-30 ; 16$ & - & - & - & - \\
\hline SMA (BA 6) & 5.19 & $6 ; 3 ; 69$ & - & - & - & - \\
\hline S2 (BA 40) L & 7.46 & $-63 ;-15 ; 12$ & 5.88 & $-63 ;-15 ; 15$ & - & - \\
\hline$S 2$ (BA 40) $R$ & 7.62 & $60 ; 12 ; 15$ & 4.63 & $60 ;-9 ; 12$ & 3.78 & $60 ;-21 ; 18$ \\
\hline Insula (BA 43) L & 4.38 & $-51 ; 6 ; 3$ & 3.43 & $-39 ; 12 ; 0$ & - & - \\
\hline Insula (BA 43) R & 5.24 & $45 ; 9 ; 0$ & 4.19 & $36 ; 21 ;-3$ & - & - \\
\hline BA $44 \mathrm{ri}$ & 5.94 & $57 ; 18 ; 12$ & - & - & 3.50 & $60 ;-9 ; 18$ \\
\hline Parietal (BA 39) L & 4.32 & $-60 ;-42 ; 19$ & 3.94 & $-57 ;-42 ; 6$ & - & - \\
\hline Parietal (BA 39) $R$ & - & - & 4.22 & $63 ;-33 ; 9$ & - & - \\
\hline CG (BA 24/32) & 5.01 & $3 ; 3 ; 42$ & 4.40 & $-6 ; 21 ; 30$ & 3.14 & $14 ;-2 ; 32$ \\
\hline Orbitofrontal (BA 10 and 47) R & 4.01 & $51 ; 24 ;-9$ & 4.18 & $51 ; 27 ;-9$ & - & - \\
\hline
\end{tabular}

during rectal and $7.5 \mathrm{ml}$ during anal stimulation; see Fig. 4).

\section{DISCUSSION}

This study for the first time describes cerebral activation during stimulation of a gastrointestinal compartment that resembles both autonomic and somatic pathways. This dual innervation pattern raises the question which of the observed activations are specific for anal and which are specific for rectal stimulation.

The S1 activation of the somatosensory cortex inbetween the hand and the foot representation as previously described by Stottrop et al. (1998) seems to be specific for anal somatosensory stimuli. Aziz et al. (2000b) concluded that visceral and somatosensory representation differentiates in the $\mathrm{S} 1$ activation since only somatosensory stimulation elicits activation in S1. The present study supports this conclusion.

Activation in motor regions was present only during anal stimulation (left M1, SMA, and cerebellum). M1 may be activated due to a voluntary-reflectory increase of the anal sphincter after distention since it was only present $6 \mathrm{~s}$ after anal stimulation and absent during the early responses before $6 \mathrm{~s}$. Activation maxima in the CG (BA 24, 32) showed topographical differences during anal and rectal stimulation. Anal stimulation elicited a medial-superior CG activation maxima, which was located more posterior than during rectal stimulation. This area is involved also in motor control (e.g., Devinsky et al., 1995; Deiber et al., 1999) and therefore, these findings may be interpreted as a re flectory motor response to somatosensory stimulation as has been described previously (Spiegel et al., 1999). Some studies have focused on the fact that during somatosensory stimuli M1 activation is present (e.g., electrical medianus stimulation; Spiegel et al., 1999). The data of the present study indicate differential activation of visceral and somatosensory representation, with somatosensory stimuli activating only areas connected to motor preparation and execution. This coactivation was not observed during rectal stimulation, probably due to the more diffuse character of the visceral stimuli in comparison to the somatosensory stimuli. The SMA and cerebellar activation sites during anal stimulation may indicate the same mechanism. The observed left hemispheric cerebellar activation maxima during anal stimulation was located lateral and inferior to the location reported by Hamdy et al. (1999) during visceromotor esophagus stimulation.

It was shown previously-by means of magnetoencephalography - that an S2 response following visceral (esophageal) stimulation was observed as early as 25 ms after stimulation onset; this allowed the authors to conclude that for visceral stimuli S2 reflects the primary response rather than S1 activation (Schnitzler et al., 1999). During somatosensory stimulation, S2 is also activated, but it follows the S1 activation after 50- 60 ms (e.g., Forss et al., 1995). Since the visceral and somatosensory S2-representations differ in the temporal domain only, the temporal resolution of fMRI does not allow us to contribute to any specificity discussion for S2 (Table 2).

Activation in the anterior left inferior parietal lobe was observed during both anal and rectal stimuli. This area represents aspects of the body schema. After lesions in the left inferior parietal lobe, predominantly movements related to the patient's own body are disturbed (Halsband et al., 2001). Artificial stimulation of the anal region-as was used here-may violate the highly habituated visceromotor pattern of internal representation with "natural" stimuli such as stool pres- 

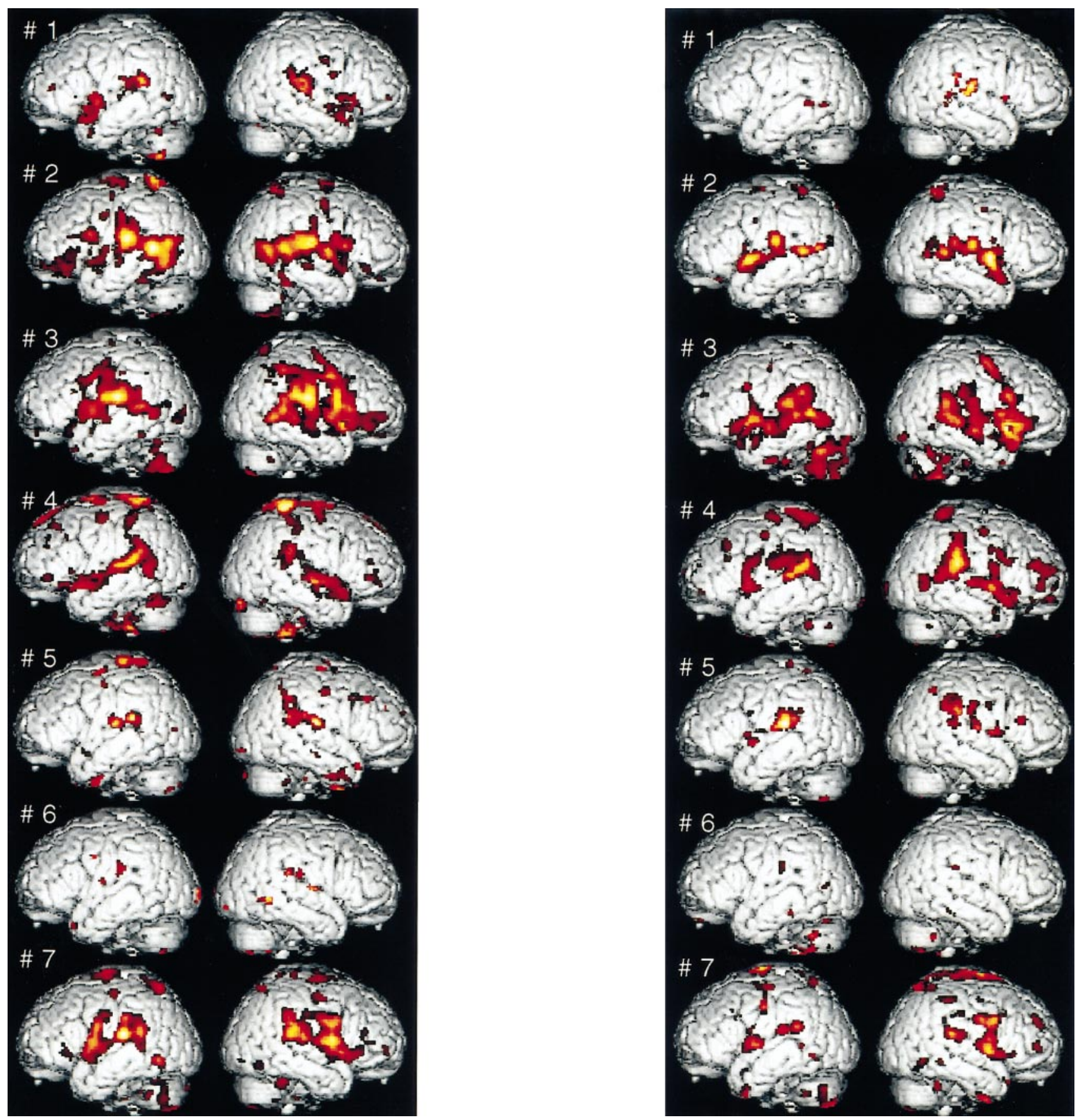

FIG. 4. Individual data for all subjects during anal (left) and rectal (right) stimulation demonstrating the variation of the activation maps $(\mathrm{P}<0.01$; uncorrected). The low cerebral activation in subject 6 may be due to a very sensitive reaction to the stimuli: air inflation of the balloon was lowest in this subject for both types of stimuli.

sure or distention after body movement. This is further supported by recent data from patients undergoing surgical removal of the rectal reservoir (Wietek et al., 2001).

Both rectal and anal stimulation evoked aversive but not pain-related emotions (4.76 to 5.24 on the 10-point aversiveness scale). In higher mammals the assessment of the emotional value of aversive stimuli encom- passes a complex neural network involving the amygdala, the anterior cingulate, and the orbitofrontal cortex (Rolls, 1999). I n the present study the amygdala and the periaqueductal gray were activated only during anal stimulation without correction for multiple comparisons. Therefore, these results need replication in independent samples. The right orbitofrontal cortex and the bilateral insula were activated during both 
TABLE 2

Specificity for Brain Regions for Anal and Rectal Stimulation

\begin{tabular}{|c|c|c|c|}
\hline Process & $\begin{array}{c}\text { Anal } \\
\text { stimulation }\end{array}$ & $\begin{array}{c}\text { Rectal } \\
\text { stimulation }\end{array}$ & Specific? \\
\hline \multirow[t]{2}{*}{ Sensory } & S1 L & - & Specific for anal \\
\hline & $\mathrm{S} 2$ bilateral & $\mathrm{S} 2$ bilateral & Not specific \\
\hline \multirow{3}{*}{ Motor } & M1 L & - & Specific for anal \\
\hline & SMA & - & Specific for anal \\
\hline & Cerebellum L & - & Specific for anal \\
\hline \multirow[t]{2}{*}{ Emotion } & Ant. insula & Ant. insula & Not specific \\
\hline & Orbitofrontal & Orbitofrontal & Not specific \\
\hline
\end{tabular}

types of stimuli. The right orbitofrontal activation seems to indicate the evaluation of aversive valence (see also Davidson et al., 2000). Aziz et al. (2000b) assumed that the processing of visceral sensations occurs in the paralimbic and limbic areas such as the insular cortex, ACG, and prefrontal cortex. Although we observed a more anterior located representation for visceral than for somatosensory stimuli, actual ACG activation was not seen in the present study. This representation site may be more specific for painful stimuli as has been demonstrated previously (for attentional changes triggered by pain see Peyron et al., 1999). Although, in the present study stimuli below pain threshold were presented, most of the activated regions during both conditions (prefrontal cortex, somatosensory cortex, inferior parietal lobe, posterior CG) were quite similar to those observed previously during painful rectal stimulation (Baciu et al., 1999). Bouras et al. (1999) assumed that anterior cingular and thalamic activations are specific for the perception of viscerorectal pain. This is supported by our data since these regions did not show significant activation during aversive anal and rectal stimuli. Both types of stimuli elicited activation in right BA 47, a region also involved in emotional processing. Blair and Cipolotti (2000) recently described a patient with lesions in this area presenting a severe difficulty in recognition of emotional expression and autonomic responding. Interestingly subtraction of anal minus rectal stimuli revealed activation in right superior BA 44, which may indicate a close relation of the representation of the upper (vocalization, swallowing, esophageal stimulation) and lower (anal somatosensory stimulation) digestive tract.

\section{SU MMARY}

We observed activation of brain areas during anorectal stimulation involved in the following processes: discriminative sensory (S1 and S2), motor responses (M1, SMA, medial CG, cerebellum), self-perception of the body schema (inferior parietal), and attention and emo- tional evaluation (CG, insula, and orbitofrontal lobe). Primary sensory and motor cortex, SMA, and right S2 activations were significantly increased during anal stimulation.

The present study confirms previous results following stimulation of the viscera at the level of the rectum and the esophagus and extends current knowledge of a "visceral" brain network with respect to somatosensory and visceral integration for a single gastrointestinal compartment which is under both autonomic and somatic-motor control. Similar to the cortical organization of swall lowing (Hamdy et al., 1999) and micturition (Nour et al., 2000), the "anorectal" brain network described here constitutes the neuronal basis of continence control and adequate elimination function, which is frequently disturbed in patients with functional or organic bowel disorders. It remains to be shown whether and how some of the demonstrated activation patterns differ in patients with peripheral lesions, at the level of both the intestine and the spinal cord, compared to functional disorders with no morphological or anatomical correlate of the abdominal complaints.

\section{ACKNOWLEDGMENTS}

This work was supported by Volkswagen-Stiftung and the Deutsche F orschungsgemeinschaft (DFG).

\section{REFERENCES}

Athwal, B. S., Berkley, K. J ., Hussain, I., Brennan, A., Craggs, M., Sakakibara, R., Frackowiak, R. S. J ., and Fowler, C. J . 2001. Brain responses to changes in bladder volume and urge to void in healthy men. Brain 124: 369-377.

Aziz, Q., Thompson, D. G., Hamdy, S., Sarkar, S., Brammer, M. J ., Bullmore, E. T., Hobson, A., Tracey, I., Gregory, L., Simmons, A., and Williams, S. C. R. 2000a. Cortical processing of human somatic and visceral sensation. J . Neurosci. 20: 2657-2663.

Aziz, Q., Schnitzler, A., and Enck, P. 2000b. Functional neuroimaging of visceral sensation. J . Clin. Neurophysiol. 17: 604-612.

Baciu, M. V., Bonaz, B. L., Papillon, E., Bost, R. A., Le Bas, J. F., Fournet, J., and Segebarth, C. M. 1999. Central processing of rectal pain: A fMRI study. Am. J . Neuroradiol. 20: 1920-1924.

Bielefeldt, K., Enck, P., and Erckenbrecht, J . F. 1990. Sensory and motor function in the maintenance of anal continence. Dis. Colon Rectum 33: 674-678.

Binkofski, F., Schnitzler, A., Stottrop, K., and Enck, P. 2000. Limbic and somatic cortex activations in electrical anorectal stimulation. Neurol mage 11(Suppl.): S893.

Blair, R. J. R., and Cipolotti, L. 2000. Impaired social response reversal: A case of 'acquired sociopathy'. Brain 123: 1122-1141.

Bouras, E. P., O'Brian, T. J ., Camilleri, M., O'Connor, M. K., and Mullan, B. P. 1999. Cerebral topography of rectal stimulation using single photon emission computed tomography. Gastrointest. Liver Physiol. 40: G687-694.

Coghill, R. C., Sang, C. N., Maisog, J . M., and I adarola, M. J . 1999. Pain intensity processing within the human brain: A bilateral distributed mechanism. J. Neurophysiol. 82: 1934-1943. 
Davidson, R. J ., Putman, K. M., and Larson, C. L. 2000. Dysfunction in the neural circuitry of emotion regulation-A possible prelude to violence. Science 289: 591-594.

Deiber, M. P., Honda, M., I banez, V., Sadato, N., and Hallett, M. 1999. Mesial motor areas in self-initiated versus externally triggered movements examined with fMRI: Effect of movement type and rate. J. Neurophysiol. 81: 3065-3077.

Devinsky, O., Morrell, M. J ., and Vogt, B. A. 1995. Contributions of anterior cingulate cortex to behavior. Brain 118: 279-306.

Di-Piero, V., Ferracuti, S., Sabatini, U., Pantano, P., Cruccu, G., and Lenzi, G. L. 1994. A cerebral blood flow study on tonic pain activation in man. Pain 56: 167-173.

Duthie, H. L., and Bennett, R. C. 1963. The relation of sensation in the anal canal to the functional anal sphincter: A possible factor in anal continence. Gut 4: 179-182.

Forss, N., J ousmaki, V., and Hari, R. 1995. Interaction between afferent input from fingers in human somatosensory cortex. Brain Res. 685: 68-76.

Goligher, J . C., and Hughes, E. S. R. 1951. Sensibility of the rectum and colon: Its role in the mechanism of anal continence. Lancet 1: 543-548.

Grodd, W., Huelsmann, E., Lotze, M., and Wildgruber, D. 2001. Mapping the cerebellum: fMRI evidence of a somatotopic organization. Hum. Brain Mapp.13: 55-73.

Halsband, U., Weyers, M., Schmitt, J ., Binkofsky, F., Grützner, G., and Freund, H.J . 2001. Recognition and imitation of pantomimed motor acts after unilateral parietal and premotor lesions: A perspective on apraxia. Neuropsychologia 39: 200-216.

Hamdy, S., Rothwell, J. C., Brooks, D. J ., Bailey, D., Aziz, Q., and Thompson, D. G. 1999. I dentification of the cerebral loci processing human swallowing with $\mathrm{H}_{2} \mathrm{O}-15$ PET activation. J . Neurophysiol. 81: 1917-1926.

Klose, U., Erb, M., Wildgruber, D., Müller, E., and Grodd, W. 1999. I mprovement of the acquisition of large amount of MR-images on a conventional whole body system. Magn. Reson. Imaging 17: 471- 474.

Mertz, H., Morgan, V., Tanner, G., Pickens, D., Price, R., Shyr, Y., and Kessler, R. 2000. Regional cerebral activation in irritable bowel syndrome and control subjects with painful and nonpainful rectal distension. Gastroenterology 118: 842- 848.
Nour, S., Svarer, C., Kristensen, J. K. I., Paulson, O. B., and Law, I. 2000. Cerebral activation during micturition in normal men. Brain 123: 781-789.

Peyron, R., Larrea, L. G., Gregoire, M. C., Costes, N., Convers, P., Lavenne, F., Mauguiere, F., Michel, D., and Laurent, B. 1999. Haemodynamic brain responses to acute pain in humans. Brain 122: $1765-1779$.

Rolls, E. T. 1999. The Brain and Emotion. Oxford Univ. Press, Oxford

Schnitzler, A., Volkmann, J ., Enck, P., Frieling, T., Witte, O., and Freund, H. J . 1999. Different cortical organization of visceral and somatic sensation in humans. Eur. J . Neurosci. 11: 305-315.

Silvermann, D. H. S., Munakata, J. A., Ennes, H., Mandelkern, M. A., Hoh, C. K., and Mayer, E. A. 1997. Regional cerebral activity in normal and pathological perception of visceral pain. Gastroenterology 112: 64-72.

Spiegel, J ., Tintera, J ., Gawehn, J ., Stoeterm, P., and Treedem, R. D. 1999. F unctional MRI of human primary somatosensory and motor cortex during median nerve stimulation. Clin. Neurophysiol. 110: 47-52.

Stottrop, K., Schnitzler, A., Witte, O. W., Freund, H. J ., and Enck, P. 1998. Cortical representation of the anal canal. Gastroenterology 114: A167.

Talairach, J ., and Tournoux, P. 1988. Co-planar Stereotaxic Atlas of the Human Brain. Thieme, Stuttgart/New York.

Tölle, T. R., Kaufmann, T., Siessmeier, T., Lautenbacher, S., Berthele, A., Munz, F., Zieglgänsberger, W., Willoch, F., Schwaiger, M., Conrad, B., and Bartenstein, P. 1999. Region-specific encoding of sensory and affective components of pain in the human brain: $A$ positron emission tomography correlation analysis. Ann. Neurol. 45: $40-47$

Whitehead, W. E., Engel, B. T., and Schuster, M. M. 1981. Perception of rectal distension is necessary to prevent fecal incontinence. In Advances in Physiological Sciences, Vol. 17, Brain and Behavior (G. Adam, I. Meszaros, and E. I. Banyai, Eds.), pp. 203-209. Akademiai Kiado, Budapest.

Wietek, B., Hinninghofen, H., Lotze, M., J ehle, E. C., Grodd, W., and Enck, P. 2001. fMRI brain mapping in patients with ulcerative colitis prior and post surgery. Digestion 63: 278. 